\title{
The quest for magnetic monopoles - past, present and future
}

\author{
Vasiliki A. Mitsou* \\ Instituto de Física Corpuscular (IFIC), CSIC - Universitat de València, \\ Parc Científic de la U.V., C/ Catedrático José Beltrán 2, \\ E-46980 Paterna (Valencia), Spain \\ E-mail: vasiliki.mitsou@ific.uv.es
}

\begin{abstract}
A short review on the state-of-the-art of searches for magnetic monopoles is presented. Theoretical scenarios predicting them and the motivation behind postulating the existence of monopoles are briefly highlighted. Various techniques for direct and indirect searches of monopoles are reviewed, whereas emphasis is given on the results of the searches and the exclusion limits set in cosmic and collider experiments. Present and future experiments for magnetic monopole detection, such as ATLAS and MoEDAL at the LHC, is also discussed.
\end{abstract}

Corfu Summer Institute 2017 'School and Workshops on Elementary Particle Physics and Gravity'

2-28 September 2017

Corfu, Greece

${ }^{*}$ Speaker. 


\section{Introduction}

The principal theoretical motivation behind the introduction of magnetic monopoles is the symmetrisation of the Maxwell's equations and the explanation of the charge quantisation [1]. Dirac showed that the mere existence of a monopole in the Universe could offer an explanation for the discrete nature of the electric charge, leading to the Dirac Quantisation Condition (DQC),

$$
\alpha g=\frac{N}{2} e, \quad N=1,2, \ldots,
$$

where $e$ is the electron charge, $\alpha=\frac{e^{2}}{4 \pi \varepsilon_{0}}=\frac{1}{137}$ is the fine structure constant, $\varepsilon_{0}$ is the vacuum permittivity, and $g$ is the monopole magnetic charge in units $\hbar=c=1$. This quantisation condition should be modified by a factor of three if free quarks are found. The monopole mass and spin remain a free parameter of the theory.

Such an appealing proposal exhilarated a number of experimental investigations since then, which are briefly reviewed here. The structure of this paper is as follows. Section 2 serves as a brief reference of the various theoretical scenarios predicting the existence of magnetic monopoles. The monopole interaction with matter and related detection techniques are discussed in Sec. 3. The state-of-the-art in monopole searches is discussed in Sec. 4 for monopoles of cosmic origin and in Sec. 5 for experiments in colliders. The paper concludes with an outlook in Section 6.

\section{Theoretical proposals}

Magnetic monopoles, carrying isolated magnetic charges, analogous to electrically-charged particles, have been hypothesised over the years in numerous theoretical proposals [1-4]. Dyons, possessing both magnetic and electric charge, pose a more complex solution to the DQC, which depends on the underlying theoretical scenario. Some of the theories predicting the existence of a magnetic monopole are highlighted below.

The Dirac monopole In Dirac's formulation [1, 5], magnetic monopoles are assumed to exist as point-like particles and quantum mechanical consistency conditions lead to Eq. (1.1), establishing the value of their magnetic charge. Although monopoles symmetrise Maxwell's equations in form, there is a numerical asymmetry arising from the $\mathrm{DQC}$, namely that the basic magnetic charge is much larger than the smallest electric charge. A magnetic monopole with a single Dirac charge $g_{\mathrm{D}}$ has an equivalent electric charge of $137 \beta e / 2$. Thus for a relativistic monopole the energy loss is around 4700 times $\left(68.5^{2}\right)$ that of a minimum-ionising electrically-charged particle.

GUT monopoles Efforts to search for magnetic monopoles have been motivated considerably since Grand Unified Theory (GUT) of strong and electroweak interactions predicted the existence of magnetic monopoles [6, 7]. In 1974, 't Hooft and Polyakov [2] pointed out that a unified gauge theory in which electromagnetism is embedded in a semi-simple gauge group, such as $S U(2)$, would predict the existence of the monopole as a soliton with spontaneous symmetry breaking. To be more specific, a semi-simple non-abelian gauge group may break into its subgroups including $U(1)$ which essentially describes magnetic monopole in 
the framework of GUT. GUT monopoles are far too massive be produced at any foreseeable human-made accelerator, having a predicted mass of $\mathscr{O}\left(10^{15} \mathrm{GeV}\right)$ or higher [8].

Global $O(3)$ monopole Global monopoles have been proposed [9] as space time (cosmological) defects allowing for the spontaneous breaking of internal global $S O(3)$ symmetries in nongauged Georgi-Glashow models. These monopoles carry no magnetic charge, however, their gravitational effects far away from their centre are significant, in the sense that the (non Minkowski) spacetime has a deficit angle. Although minute, such an effect affects the forward scattering amplitude of Standard Model (SM) particles that propagate in such backgrounds, leading to ring-like angular regions, where the scattering amplitude is very large [10]. Such peculiar scattering patterns of ordinary SM particles may indicate indirectly the presence of a neutral global monopole in collider detectors, where they may pairproduced $[9,11]$. Furthermore, a variant of the global monopole model, including axion fields (with a given "axion charge") and a real electromagnetic field, which couples only gravitationally to the scalar $S O(3)$ symmetry breaking sector, has been considered [12], resulting into axions capable of inducing electromagnetic monopole solutions with a real magnetic charge of order of the "axion charge". In such a case, both the high ionisation and the peculiar effects [10] of the monopole background on the scattering of ordinary particles on them are in operation.

Electroweak monopole The electroweak monopole, proposed by Cho and Maison [4, 13], is a generalisation of the Dirac monopole, representing a hybrid of Dirac and 't Hooft-Polyakov monopoles that carries magnetic charge twice that of the Dirac monopole. This is because it is based on the quotient group $S U(2) \otimes U_{Y}(1) / U_{\mathrm{em}}(1)$, where $U_{\mathrm{em}}(1)$ is the (unbroken) group of electromagnetism instead of, e.g., the $S U(2)$ group in the Georgi-Glashow model. Recent estimates of the electroweak monopole mass [14] indicate that it is possibly detectable at the LHC.

Monopolium A possible explanation for the lack of experimental confirmation of monopoles is Dirac's proposal $[1,5,15]$ that monopoles are not seen freely because they form a bound state called monopolium [16, 17] being confined by strong magnetic forces. Monopolium is a neutral state, hence it is difficult to detect directly at a collider detector, although its decay into photons would give a rather clear signal for the ATLAS, CMS and CT-PPS detectors [18, 19].

\section{Detection techniques}

The detection techniques for monopoles rely on their interactions with matter. Different monopole-matter interactions are briefly discussed in this section. The same technique may be applied for monopoles of different origin, i.e. either for cosmic rays or produced in colliders.

\subsection{Ionisation and excitation}

Any charged particle carrying electromagnetic charge will deposit some amount of energy by the ionisation process and excitation of atoms when it is moving in matter. Thus scintillator, 
gas detectors, and nuclear track detectors (NTDs), are sensitive to monopoles, as they all fall into this category of detection technology. To calculate the energy loss of monopoles passing through matter, we need to consider how the energy of the monopole is transferred to the surrounding medium. There are basically three ways where the energy can be dissipated and their importance depends on the monopole velocity $\beta$ and the medium.

Ionisation The energy is transferred to the production of free electrons. This is well described by the Bethe-Bloch formula discussed below and is the dominant energy-loss mechanism for fast monopoles $(\beta>0.05)$ moving in gaseous detectors.

Atomic excitation The energy is transferred to atoms of higher energy states. It starts to dominate the energy loss at slower monopole speeds, i.e. $10^{-3} \lesssim \beta \lesssim 10^{-2}$.

Elastic collisions with atoms The energy loss is due to atoms (nuclei) recoiling through the monopole coupling to atomic or nuclear magnetic moment. It becomes important for even slower monopoles $\left(\beta \lesssim 10^{-3}\right)$ and different energy-loss calculations have been performed for diamagnetic and paramagnetic materials.

The energy loss per path length travelled, $\mathrm{d} E / \mathrm{d} x$, due to ionisation for a particle with electric charge $z e$ is given by the Bethe-Bloch formula [20]

$$
-\frac{\mathrm{d} E}{\mathrm{~d} x}=z^{2} \frac{K Z}{A} \frac{1}{\beta^{2}}\left[\frac{1}{2} \ln \left(\frac{2 m_{\mathrm{e}} \beta^{2} \gamma^{2}}{I^{2}}\right)-\beta^{2}\right],
$$

where $K=4 \pi N_{\mathrm{A}} r_{\mathrm{e}}^{2} m_{\mathrm{e}} c^{2}$, with $Z(A)$ the atomic number (mass) of the medium and $I$ its excitation energy; $m_{\mathrm{e}}\left(r_{\mathrm{e}}\right)$ the electron mass (radius); and $N_{\mathrm{A}}$ the Avogadro number. The ionisation energy loss for electric charges can be adapted for a magnetic charge $n g_{\mathrm{D}}$ by replacing $z e \rightarrow n g_{\mathrm{D}} \beta$ [21]. The stopping power then becomes

$$
-\frac{\mathrm{d} E}{\mathrm{~d} x}=\left(n g_{\mathrm{D}} / e\right)^{2} \frac{K Z}{A}\left[\frac{1}{2} \ln \left(\frac{2 m_{\mathrm{e}} \beta^{2} \gamma^{2}}{I^{2}}\right) \cdot-\beta^{2}\right]
$$

For instance, a relativistic monopole with charge $g_{\mathrm{D}}$ loses energy as a nucleus with $z \simeq 69$, or $\left(g_{\mathrm{D}} / e\right)^{2}=68.5^{2} \simeq 4700$ times more than an electron. This makes even a singly-charged monopole a highly-ionising particle.

Through the ionisation process and excitation of atoms, liquid or plastic scintillators, gas detectors (e.g. drift or streamer tubes), and NTDs, are sensitive to monopoles. One disadvantage of this detection technique is that it is in general difficult to disentangle magnetic from electric charge solely from the energy deposit information. So a careful review of the theoretical models is necessary to predict the energy loss of monopole through matter and the light yield in the scintillator. And care must be taken in the analysis of the data to eliminate all other possible sources of such signatures.

\subsection{Induction}

This detection technique is based on the long-range electromagnetic interaction of a monopole with the microscopic state of a superconducting loop and it is directly sensitive to the magnetic charge $g$. The magnetic flux of a monopole passing through the loop is given by $4 \pi g=h c / e$, where $h$ in the Planck constant and $c$ the speed of light. A superconducting loop compares this flux with the elementary flux quantum $\phi_{0}=h c / 2 e$, where the factor two arises from the electrons 
appearing as Cooper pairs. The induced persistent electric current $\Delta i$ in a coil with $N$ turns and inductance $L$ is given by the formula

$$
\Delta i=4 \pi N g / L
$$

The major background for these experiments are small changes in Earth's magnetic field, therefore shielding of the ambient field is required with extreme caution, leading to high costs for detectors with broad surveillance areas. As a consequence, this technique is no longer used to search for monopoles of cosmic origin, yet it is still widely used in searches for monopoles bound in matter.

\section{3 Çerenkov light}

When traversing a medium such as water or ice, relativistic monopoles would lose some of their energy to Çerenkov radiation. When the monopole speed exceeds the group velocity of light in that particular medium, photons are emitted from excited atoms in the medium. Electrically charged particles also give rise to Çerenkov radiation, yet the number of photons emitted is then much smaller. In water and ice, having a refractive index $n_{\mathrm{r}} \simeq 1.33$, a monopole with one Dirac charge generates $\left(g_{\mathrm{D}} n_{\mathrm{r}} / e\right)^{2} \simeq 8300$ more photons than a particle with one electric unit charge traveling with the same speed. The radiation can only be produced by particles with speeds above a threshold of $\beta_{\mathrm{thr}}=1 / n_{\mathrm{r}} \simeq 0.75$. The photons are emitted coherently under a fixed angle $\cos \theta_{\mathrm{C}}=$ $1 / \beta n_{\mathrm{r}}$, which for water or ice is $\theta_{\mathrm{C}} \simeq 41.2^{\circ}$ for relativistic monopoles.

\subsection{Catalysis of nucleon decay}

It has been proposed that the boson in the core of a GUT monopole may cause nucleons to decay by performing transitions between quarks and leptons, as predicted by the Callan-Rubakov mechanism [22]. Such processes, such as $u u d \rightarrow e^{+} \bar{d} d$ and $u d d \rightarrow e^{+} \bar{u} d$, violate the baryonnumber conservation. The process cross section, $\sigma_{0}$, is of the same order as that of the strong interactions while the branching fractions of the aforesaid transitions exceed $90 \%$.

The decay products, being much lighter than their parents, are highly relativistic. Therefore, along the trajectory of a catalysing monopole in medium, outbursts of Çerenkov radiation would occur. The catalysis cross section depends on the monopole-nucleon relative velocity as $\sigma_{\text {cat }}=$ $\left(\sigma_{0} / \beta\right) F(\beta)$, where $F(\beta)$ is a correction factor relevant for speeds below a threshold. Hence nucleon-decay catalysis allows detection of extremely massive sub-relativistic monopoles.

\section{Searches for monopoles of cosmic origin}

If magnetic monopoles of cosmic origin do exist, they must have been formed shortly after the Big Bang, presumably as topological defects arising when the Universe expanded and cooled. The existence of the galactic magnetic field $B \simeq 3 \mu \mathrm{G}$ would accelerate such monopoles, thus draining energy from the magnetic field. In order for the galactic field to sustain, its dissipation must not exceed its regeneration. This requirement implies that an upper flux limit should be respected, the so-called Parker bound [23]

$$
\Phi \lesssim 10^{-15} \mathrm{~cm}^{-2} \mathrm{~s}^{-1} \mathrm{sr}^{-1}
$$


The (tighter) extended Parker bound takes into account the survival of a small galactic seed field and lowers the flux bound to [24]:

$$
\Phi \lesssim 10^{-16}\left(m_{\mathrm{M}} / 10^{17} \mathrm{GeV}\right) \mathrm{cm}^{-2} \mathrm{~s}^{-1} \mathrm{sr}^{-1}
$$

Blas Cabrera and collaborators at Stanford University set up an experiment with a four-turn, 5-cm-diameter loop, positioned with its axis vertical, connected to the superconducting input coil of a SQUID (superconducting quantum interference device) magnetometer [25]. He reported a single candidate event during the 151 days of running of his experiment on February $14^{\text {th }} 1982$. If this candidate event is considered to be spurious, his data set an upper limit of $6.1 \times 10^{-10} \mathrm{~cm}^{-2} \mathrm{~s}^{-1} \mathrm{sr}^{-1}$, which is much larger than the Parker bound. Despite further improvements of the experimental setup to suppress possible background sources, this result was not confirmed. The best bound given by an induction detector on cosmic monopoles was obtained later by the same group: $90 \%$ CL limit on monopole flux of $7.2 \times 10^{-10} \mathrm{~cm}^{-2} \mathrm{~s}^{-1} \mathrm{sr}^{-1}$ [26].

Another monopole candidate had been seen earlier in 1973 at Berkeley by a team led by P. B. Price, in a balloon-borne experiment. The NTD sheets had recorded an unusual track corresponding to a highly-ionising particle moving downward at relativistic speed [27]. The track seemed consistent with a magnetic monopole of charge $g_{\mathrm{D}}$ and a mass of $\lesssim 200 \mathrm{GeV}$. However, thorough studies indicated that the track was probably due to the fragmentation of a platinum nucleus.

Monopoles of cosmic origin can be detected by exploiting any of the techniques outlined in Sec. 3. The search may involve old material where monopoles may be either trapped or may have left tracks, as discussed in Sec. 4.1. However, most of the searches, described in Sec. 5.2, seek monopoles in-flight interacting with detectors covering a wide spectrum of monopole masses and velocities. Lastly, searches for effects of monopole-catalysed decays, e.g. proton decay, are discussed in Sec. 4.3.

\subsection{Searches for monopoles bound or having left traces in matter in the past}

The induction technique has also been deployed in searches for monopoles bound in matter, such as lunar rocks, meteorites, seawater, iron ores and manganese nodules, by passing samples through superconducting loops. With this method, a stringent upper limit on the monopoles per nucleon ratio of $\sim 10^{-29}$ has been obtained [28]. Moreover, as we shall see in Sec. 5.4, SQUIDs have been used in experiments to look for monopoles produced in high-energy collisions and trapped in $\mathrm{Al}$ and/or Be volumes.

Another sort of experiment relies on ionisation to look for traces of traversing monopoles in ancient $\left(4.6 \times 10^{8} \mathrm{yr}\right)$ mica. The analysis involves a search for defects in the molecular structure of the material caused by the propagation of a monopole in a similar way as for plastic NTDs. The observed absence of monopole tracks in the mica detector placed an upper limit of $10^{-17}$ to $10^{-16} \mathrm{~cm}^{-2} \mathrm{~s}^{-1} \mathrm{sr}^{-1}$ on the flux of GUT monopoles having velocity $\beta \sim 3 \times 10^{-4}-1.5 \times 10^{-3}$ [29]. This was the first direct search for monopoles with adequate sensitivity to detect a flux as small as the Parker flux limit. 


\subsection{Direct cosmic searches}

Such searches have been performed on underground, surface and balloon-borne experiments targeting GUT monopoles spanning masses of $100-10^{4} \mathrm{TeV}$ with a velocity range of $\beta \sim 10^{-4}-1$. As of to date, there is no experimental evidence for cosmic magnetic monopoles, only bounds on their flux as a function of mass and velocity. Present limits are shown in Fig. 1 [30].

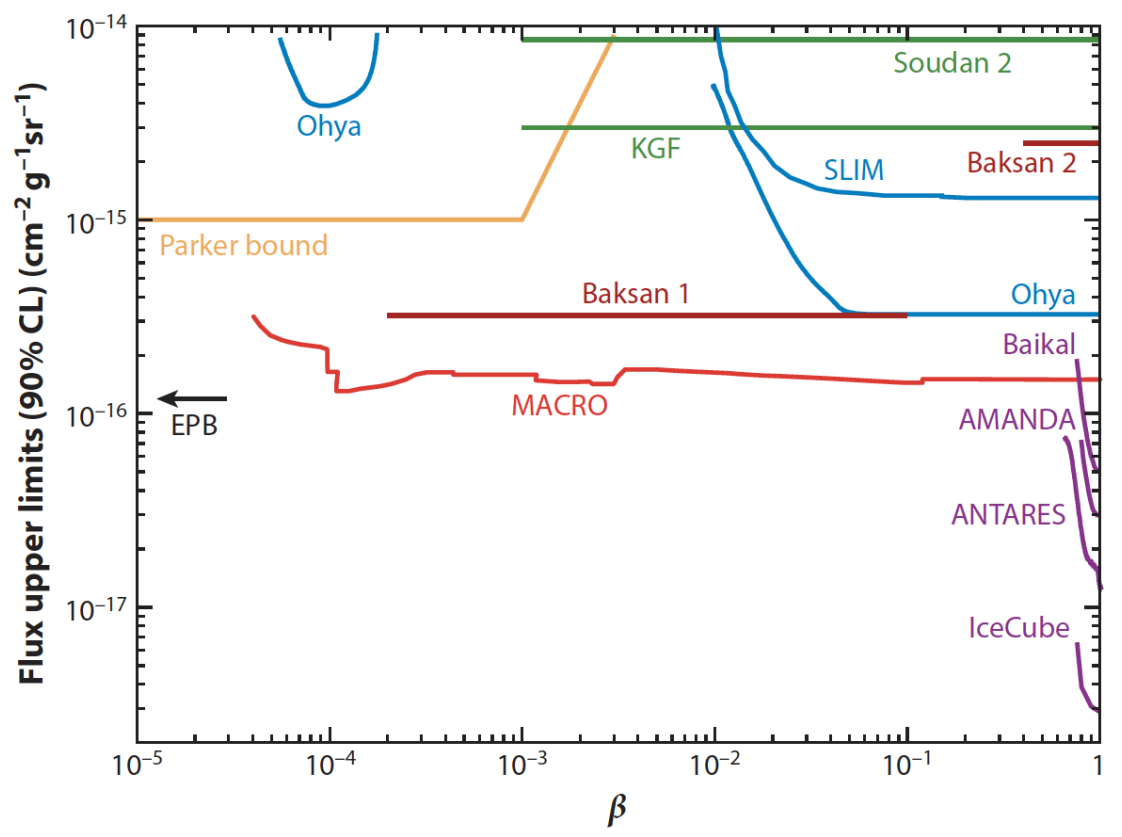

Figure 1: 90\% CL upper limits versus velocity $\beta$ for a flux of cosmic GUT monopoles with magnetic charge of $g=g_{\mathrm{D}}$. The references for the results shown are given in the text for each experiment. The Parker bound is given in Eq. (4.1), whereas the extended Parker bound (EPB) refers to Eq. (4.2). From Ref. [30].

MACRO [31] was a large underground detector located in the Gran Sasso laboratory. It has the best limits for super heavy GUT monopoles with a sensitivity that covers most of the phase space in Fig. 1, mostly thanks to the redundancy and complementarity of the various detector components it was comprising: liquid scintilation counters; limited streamer tubes; and NTDs. However, due to its underground location, it was not sensitive to lower-energy monopoles, which are blocked by the Earth. As shown in Fig. 1, the upper limit to the local monopole flux set is $1.4 \times 10^{-16} \mathrm{~cm}^{-2} \mathrm{~s}^{-1} \mathrm{sr}^{-1}$, i.e. well below the Parker bound in almost all the $\beta$ range for GUT monopoles [32]. Also shown are limits from an experiment at the Ohya stone quarries in Japan [33], which used a $2000 \mathrm{~m}^{2}$ array of CR39 ${ }^{\circledR}$ NTDs. Another experiment at Baksan [34] in Russia used liquid scintillation counters. Soudan 2 [35], in the United States, was a large, fine-grained tracking calorimeter composed of long drift tubes, and another tracking calorimeter, Kolar Gold Fields (KGF) [36], was deployed in India.

The SLIM detector, on the other hand, installed at high altitude at the Mt Chacaltaya laboratory in Bolivia with an elevation of $5400 \mathrm{~m}$, probed a region for intermediate-mass monopoles $\left(10^{5} \lesssim\right.$ $M \lesssim 10^{12} \mathrm{GeV}$ ), well below the GUT scale, which do not have enough energy to penetrate the entire atmosphere. The SLIM NTDs array covered an area $>400 \mathrm{~m}^{2}$ that after four years of exposure no signal of magnetic monopoles was observed and set limits [37] as shown in Fig. 1. 
Relativistic monopoles can be sought by the emittance of Çerenkov radiation, when traveling through a homogeneous and transparent medium such as water or ice, which can be detected by arrays or strings of photomultiplier tubes. Neutrino telescopes such as Baikal [38], AMANDA [39], ANTARES [40, 41], IceCube [42, 43], and the future KM3NeT [44] and PINGU [45] are sensitive to the huge quantity of visible Çerenkov light emitted by a monopole with $\beta>0.75$ (direct Çerenkov). Additional light is produced by Çerenkov radiation from $\delta$-ray electrons along the monopole path for velocities down to $\beta=0.625$ (indirect Çerenkov). Furthermore luminescence may be induced by molecular excitation of the medium for monopole velocities of $\beta>0.01$. Results for relativistic monopoles from MACRO [32], ANTARES [41] and IceCube [43, 46] are depicted in Fig. 2. As is the case for neutrinos, a large background from cosmic muons inhibits searches for down-going candidates; up-going monopoles having traversed the Earth before reaching the detector are probed instead. It is worth noting that GUT supermassive monopoles are unlikely to reach (nearly) relativistic velocities.

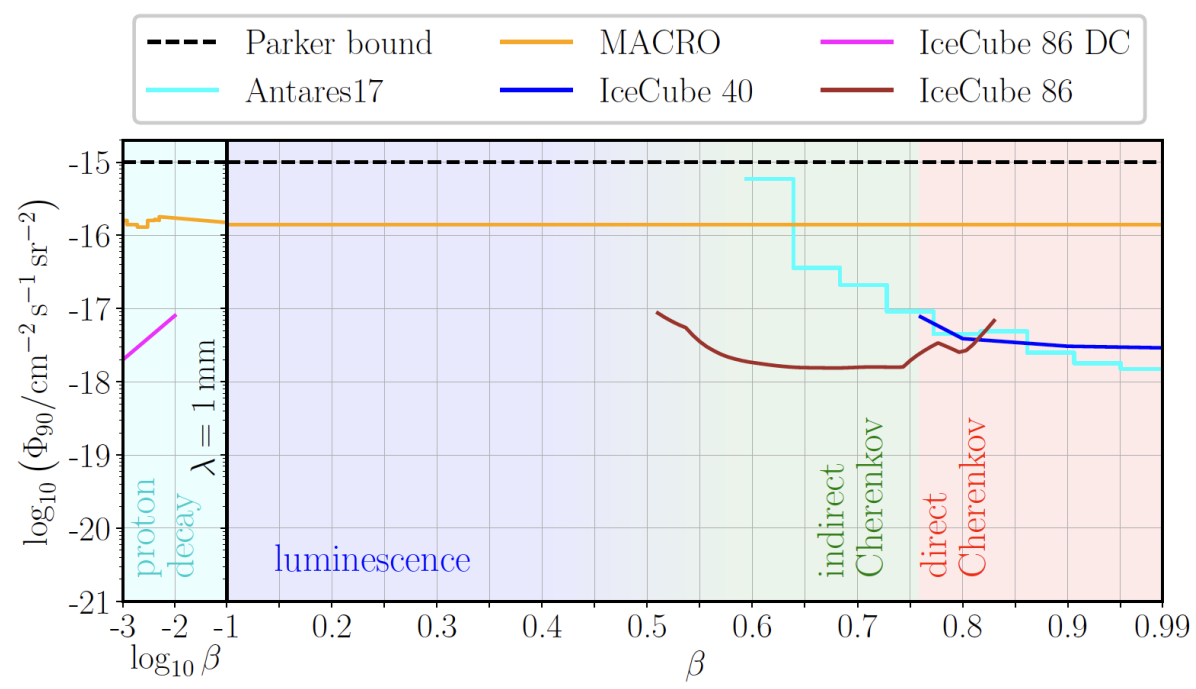

Figure 2: $90 \%$ CL upper limits versus velocity $\beta$ for a flux of very energetic cosmic GUT monopoles with magnetic charge of $g=g_{\mathrm{D}}$. The IceCube $86 \mathrm{DC}$ nucleon-decay analysis is based on the IceCube DeepCore [46]. Results from MACRO [32] and ANTARES [41] are superimposed on IceCube limits from direct (IceCube 40) and indirect (IceCube 86) Çerenkov light [43]. The Parker bound is given in Eq. (4.1). From Ref. [47].

The flux of ultra-relativistic monopoles has been constrained by the Pierre Auger Observatory, which was sensitive to monopoles with Lorentz factor values $\gamma \sim 10^{9}-10^{12}$, leading to flux limits in the range $10^{-15}-2.5 \times 10^{-21} \mathrm{~cm}^{-2} \mathrm{~s}^{-1} \mathrm{sr}^{-1}$ [48]. Two other experiments exploited the radiowave pulses from the interactions of a primary particle with ice to search for monopoles. The Radio Ice Cherenkov Experiment (RICE), consisting of radio antennas buried in the Antarctic ice, set a flux upper limit of $10^{-18} \mathrm{~cm}^{-2} \mathrm{~s}^{-1} \mathrm{sr}^{-1}$ at $95 \% \mathrm{CL}$ for intermediate-mass monopoles with $10^{7}<\gamma<10^{12}$ and a total energy of $10^{16} \mathrm{GeV}$ [49]. The ANITA-II balloon-borne radio interferometer, on the other hand, set a 90\%-CL flux upper limit on the order of $10^{-19} \mathrm{~cm}^{-2} \mathrm{~s}^{-1} \mathrm{sr}^{-1}$ for a Lorentz factor $\gamma>10^{10}$ at a total energy of $10^{16} \mathrm{GeV}$ [50]. 


\subsection{Searches through catalysis of nucleon decay}

Signals of a monopole-induced decay of a nucleon, as predicted by the Callan-Rubakov mechanism [22], have been sought, which are sensitive to the assumed value of the catalysed-decay cross section. Searches have been made with the Soudan [51] and MACRO [52] experiments, using tracking detectors. Searches at the Irvine-Michigan-Brookhaven detector (IMB) [53], the underwater Lake Baikal experiment [54] and the IceCube experiment [46] which exploit the Çerenkov effect have also been made. The resulting $\beta$-dependent flux limits from these experiments typically lie in the range $10^{-18}-10^{-14} \mathrm{~cm}^{-2} \mathrm{~s}^{-1} \mathrm{sr}^{-1}$. A recent search for low energy neutrinos, assumed to be produced from induced proton decay in the Sun, was made at Super-Kamiokande [55]. A model- and $\beta$-dependent of limit of $6.3 \times 10^{-24}(0.001 \beta)^{2} \mathrm{~cm}^{-2} \mathrm{~s}^{-1} \mathrm{sr}^{-1}$ was obtained.

\section{Searches in colliders}

Present and proposed future accelerators feature a centre-of-mass energy of $\mathscr{O}(10 \mathrm{TeV})$, thus it is impossible to search for GUT monopoles in these machines. Nevertheless, searches have been carried out to detect direct or indirect signals of lower-mass monopoles. Searches have been performed at hadron-hadron, electron-positron and lepton-hadron experiments, mostly directly using scintillation counters, gas chambers and NTDs, taking advantage of the monopole high ionisation power. Other analyses focus on exposed material for trapped monopoles or peculiar magneticcharge trajectories. In addition, virtual-monopole processes enhancing production rates of certain final states have also been considered as indirect probes for monopoles.

\subsection{Virtual monopoles}

Virtual monopoles have been suggested to mediate processes giving rise to multi-photon final states [56, 18]. Photon-based searches have been carried out by D0 [57] at the Tevatron and L3 [58] at LEP. The D0 analysis led to spin-dependent lower mass limits of between 610 and $1580 \mathrm{GeV}$, whilst $\mathrm{L} 3$ reported a lower mass limit of $510 \mathrm{GeV}$. However, the uncertainties of the cross-section calculations used to derived these limits are difficult to estimate [59]. Another interesting idea yielding a multi-photon signature is the possible production and decay or annihilation to multiple photons of the monopolium $[18,19]$.

\subsection{Past direct searches}

Collider experiments typically express their results in terms of upper limits on a production cross section versus the monopole mass. To calculate these limits, an ansatz is used to model the kinematics of monopole-antimonopole pair production processes since perturbative field theory cannot be used to calculate the rate and kinematic properties of produced monopoles [20]. Limits therefore suffer from a degree of model-dependence, implying that a comparison between the results of different experiments can be problematic, in particular when this concerns excluded mass regions. This situation may be resolved if thermal production in heavy-ion collisions - that does not rely on perturbation theory- is considered [60].

Searches for monopoles at the Tevatron have been carried out by the CDF [61] and E882 [62] experiments. The CDF experiment used a dedicated time-of-flight system whereas the E882 experiment employed the induction technique to search for stopped monopoles in discarded material 
which had been part of the CDF and D0 detectors exposed to $p \bar{p}$ collisions. Earlier searches at the Tevatron, such as at the D0 collision point [63], used NTDs and were based on comparatively modest amounts of integrated luminosity. Lower energy hadron-hadron experiments have employed a

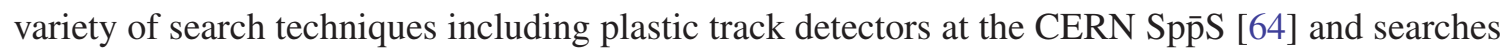
for trapped monopoles in 300-GeV-proton beam dump [65].

The only LEP-2 search was made by OPAL [66] which quoted cross section limits for the production of monopoles with masses up to around $103 \mathrm{GeV}$. At LEP-1, searches were made with NTDs deployed around an interaction region, thus allowing high charges to be probed for masses up to $\sim 45 \mathrm{GeV}$. Specifically, the L6-MODAL experiment [67] set limits for monopoles with charges in the range $0.9-3.6 g_{\mathrm{D}}$, whilst an earlier search by the MODAL experiment was sensitive to monopoles with charges as low as $0.1 g_{\mathrm{D}}$ [68]. The deployment of NTDs around the beam interaction point was also used at earlier $e^{+} e^{-}$colliders such as KEK by TRISTAN [69] and PETRA [70] at DESY. Searches at $e^{+} e^{-}$facilities have also been made for particles following non-helical trajectories with the CLEO [71] and TASSO [72] detectors.

There has so far been one search for monopole production in lepton-hadron scattering. Using the induction method, monopoles were sought which could have stopped in the Al beam pipe which had been used by the H1 [73] experiment at HERA. Cross section limits were set for monopoles with charges in the range $1-6 g_{\mathrm{D}}$ for masses up to $\sim 140 \mathrm{GeV}$.

Searches for monopoles produced at the highest available energies in hadron-hadron collisions are being carried out in $p p$ collisions at the CERN Large Hadron Collider (LHC) [74] by the ATLAS [75] and MoEDAL [76] experiments utilising different detection techniques, as discussed in Sec. 5.3 and Sec. 5.4, respectively.

\subsection{Searches for monopoles with ATLAS}

At the ATLAS experiment [75], searches for magnetic monopoles have been performed on 7-TeV [77] on 8-TeV [78] data using the transition radiation tracker (TRT) [79] sensitivity to highionisation signals. The $8-\mathrm{TeV}$ analysis relies on a dedicated trigger for highly-ionising particles, which makes use of two $\mathrm{d} E / \mathrm{d} x$ variables: the fraction of TRT hits passing a predefined high threshold (HT), $f_{\mathrm{HT}}$, and the number of hits in the TRT passing the HT, $N_{\mathrm{HT}}$. The discriminating particle characteristics used by this search are the cluster energy, the energy dispersion in the electromagnetic calorimeter, $w$, and the $f_{\mathrm{HT}}$. The energy dispersion measures the fraction of the cluster energy contained in the most energetic cells of a cluster in each of the layers of the electromagnetic calorimeters. Variables $w$ and $f_{\mathrm{HT}}$ are also used to estimate the background in a data-driven way. Figure 3 (left) shows the 2D distribution of $w$ and $f_{\mathrm{HT}}$ for data and a signal with $1 g_{\mathrm{D}}$ [78]. It is evident that the variables are well discriminating and thus can be used to predict the number of background events in the signal region (region A). Since $w$ and $f_{\mathrm{HT}}$ are not fully uncorrelated for smaller values of $w$, the control regions B and $\mathrm{D}$ are defined with a lower bound of $w \sim 0.8$.

Since no excess of data events is observed, the search is interpreted assuming the Drell-Yan (DY) production process with modified electromagnetic couplings as seen in Fig. 3 (right) [78]. The analysis is sensitive to magnetic charges of $0.5 g_{\mathrm{D}} \leq|g| \leq 1.5 g_{\mathrm{D}}$ and set limits for spin- 0 and spin-1/2 monopoles. The search excludes monopoles with a magnetic charge of $1 g_{\mathrm{D}}$ up to masses of $1340 \mathrm{GeV}$ for a spin-1/2 hypothesis of the particle. In addition, a model independent cross section upper limit of $0.5 \mathrm{fb}$ is set in fiducial regions where the selection efficiency is almost constant. 

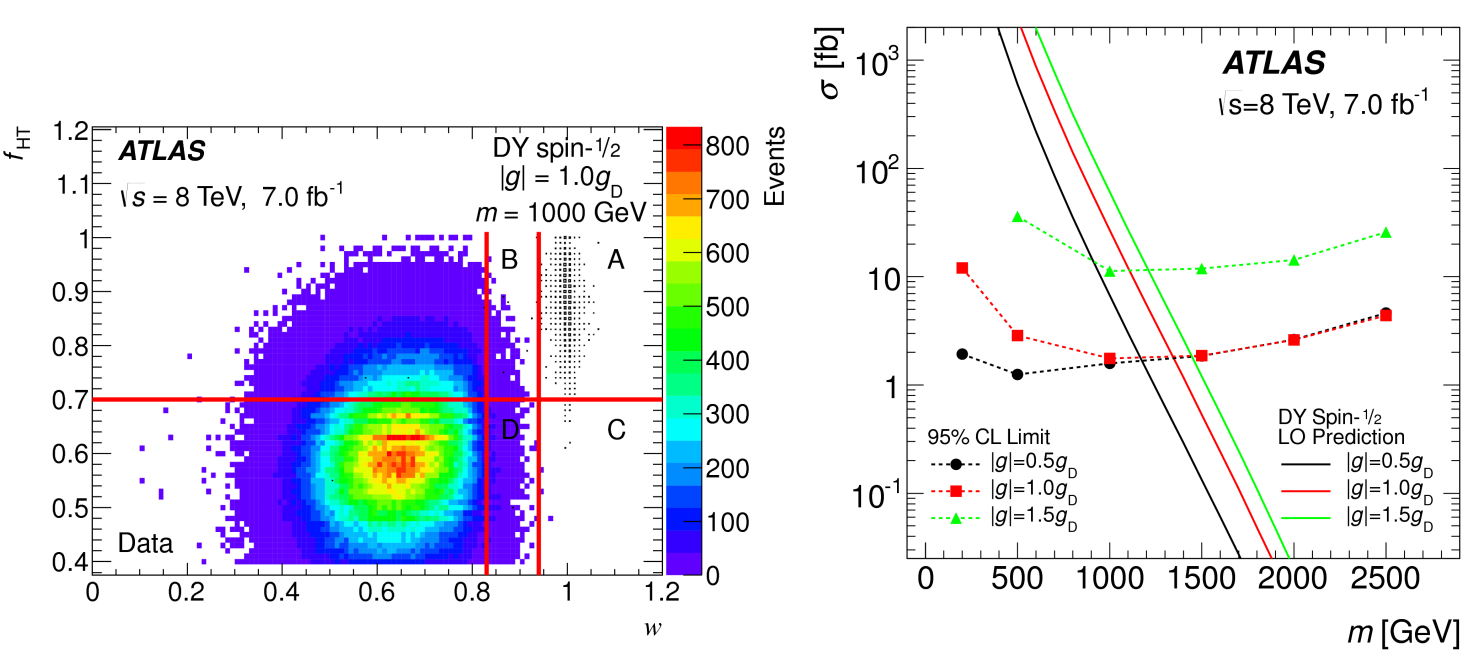

Figure 3: Left: 2D distribution of $w$ and $f_{\mathrm{HT}}$ for data (colourful) and a signal model (grey) with a spin- $1 / 2$ magnetic monopole of $1 \mathrm{TeV}$ mass. The red lines indicate the signal (A) and the control regions (B, C, D). Right: $95 \%$ cross-section upper limits (dashed lines) and theoretical cross sections (solid lines) for magnetic monopoles with spin $1 / 2$ and various magnetic charges. From Ref. [78].

\subsection{The MoEDAL experiment at the LHC}

MoEDAL (Monopole and Exotics Detector at the LHC) [76] is designed to search for manifestations of new physics through highly-ionising (HI) particles in a manner complementary to ATLAS and CMS [80]. The main motivation for the MoEDAL experiment is to pursue the quest for magnetic monopoles at LHC energies. Nonetheless the detector is also designed to search for any massive, long-lived, slow-moving particles [81] with single or multiple electric charges arising in many scenarios of physics beyond the SM [82].

\subsubsection{The MoEDAL detector}

The MoEDAL detector [76, 82] is deployed around the intersection region at Point 8 of the LHC in the LHCb experiment Vertex Locator cavern. It is a unique and largely passive LHC detector comprised of four sub-detector systems.

Nuclear track detectors The main sub-detector system is made of a large array of CR39 ${ }^{\circledR}$, Makrofol ${ }^{\circledR}$ and Lexan ${ }^{\circledR}$ NTD stacks surrounding the intersection area. The passage of a HI particle through the plastic detector is marked by an invisible damage zone along the trajectory. The damage zone is revealed as a cone-shaped etch-pit when the plastic detector is chemically etched. Then the sheets of plastics are scanned looking for aligned etch pits in multiple sheets. The MoEDAL NTDs have a threshold of $Z / \beta \sim 5$, where $Z$ is the charge and $\beta=v / c$ the velocity of the incident particle.

Another type of NTD installed is the Very High Charge Catcher $(Z / \beta \sim 50)$. It consists of two flexible low-mass stacks of Makrofol ${ }^{\circledR}$, deployed in the $\mathrm{LHCb}$ acceptance between RICH1 and the Trigger Tracker. It is the only NTD (partly) covering the forward region, 
adding only $\sim 0.5 \%$ to the $\mathrm{LHCb}$ material budget while enhancing considerably the overall geometrical coverage of MoEDAL.

Magnetic trappers A unique feature of the MoEDAL detector is the use of paramagnetic magnetic monopole trappers (MMTs) to capture magnetically-charged HI particles. The aluminium absorbers of MMTs are subject to an analysis looking for magnetically-charged particles at a remote SQUID magnetometer facility [83]. For the 2015 run at $13 \mathrm{TeV}$, the MMT consisted of 672 aluminium rods for a total mass of $222 \mathrm{~kg}$ that were placed $1.62 \mathrm{~m}$ from the IP8 LHC interaction point under the beam pipe on the side opposite to the $\mathrm{LHCb}$ detector.

TimePix radiation monitors The only non-passive MoEDAL sub-detector is an array of TimePix pixel devices distributed throughout the MoEDAL cavern, forming a real-time radiation monitoring system of $\mathrm{HI}$ beam-related backgrounds. The operation in time-over-threshold mode allows a 3D mapping of the charge spreading in the volume of the silicon sensor, thus differentiating between various particles species from mixed radiation fields and measuring their energy deposition.

\subsubsection{Searches for monopoles in MoEDAL}

The high ionisation of slow-moving magnetic monopoles and dyons, implies quite characteristic trajectories when such particles interact with the MoEDAL NTDs, which can be revealed during the etching process $[76,82]$. In addition, the high magnetic charge of a monopole (which is expected to be at least one Dirac charge $g_{\mathrm{D}}=68.5 e$ ( $c f$. Eq. (1.1)) implies a strong magnetic dipole moment, which in turn may result in a strong binding of the monopole with the ${ }_{13}^{27} \mathrm{Al}$ nuclei of the aluminium MoEDAL MMTs. In such a case, the presence of a monopole trapped in an aluminium bar of an MMT would be detected through the existence of a persistent current, defined as the difference between the currents in the SQUID of a magnetometer before and after the passage of the bar through the sensing coil.

In the context of the MMT exposure during Run 2, no magnetic charge exceeding $0.5 g_{\mathrm{D}}$ was detected in any of the exposed samples when passed through the ETH Zurich SQUID facility, allowing limits to be placed on monopole production. Model-independent cross-section limits have been obtained in fiducial regions of monopole energy and direction for $1 g_{\mathrm{D}} \leq|g| \leq 6 g_{\mathrm{D}}$ with the 8-TeV analysis [84]. Model-dependent cross-section limits are obtained for DY pair production of spin-1/2 and spin-0 monopoles for $1 g_{\mathrm{D}} \leq|g| \leq 5 g_{\mathrm{D}}$ at $13 \mathrm{TeV}$ [85], as shown in Fig. 4 (left). MoEDAL has extended these bounds including the 2016 MMT exposure, also setting the first limits for spin-1 monopoles [86].

Under the assumption of DY cross sections, mass limits are derived for $1 g_{\mathrm{D}} \leq|g| \leq 4 g_{\mathrm{D}}$ with MoEDAL [85], complementing previous results from ATLAS presented in Sec. 5.3, as shown in Fig. 4 (right). The ATLAS bounds are better that the MoEDAL ones for $|g|=1 g_{\mathrm{D}}$ due to the higher luminosity delivered in ATLAS and the loss of acceptance in MoEDAL for small magnetic charges. On the other hand, higher charges are difficult to be probed in ATLAS due to the limitations of the electromagnetic-calorimeter-based level-1 trigger deployed for such searches. A comparison of the limits on monopole production cross sections set by other colliders with those set by MoEDAL is presented in Ref. [7]. 

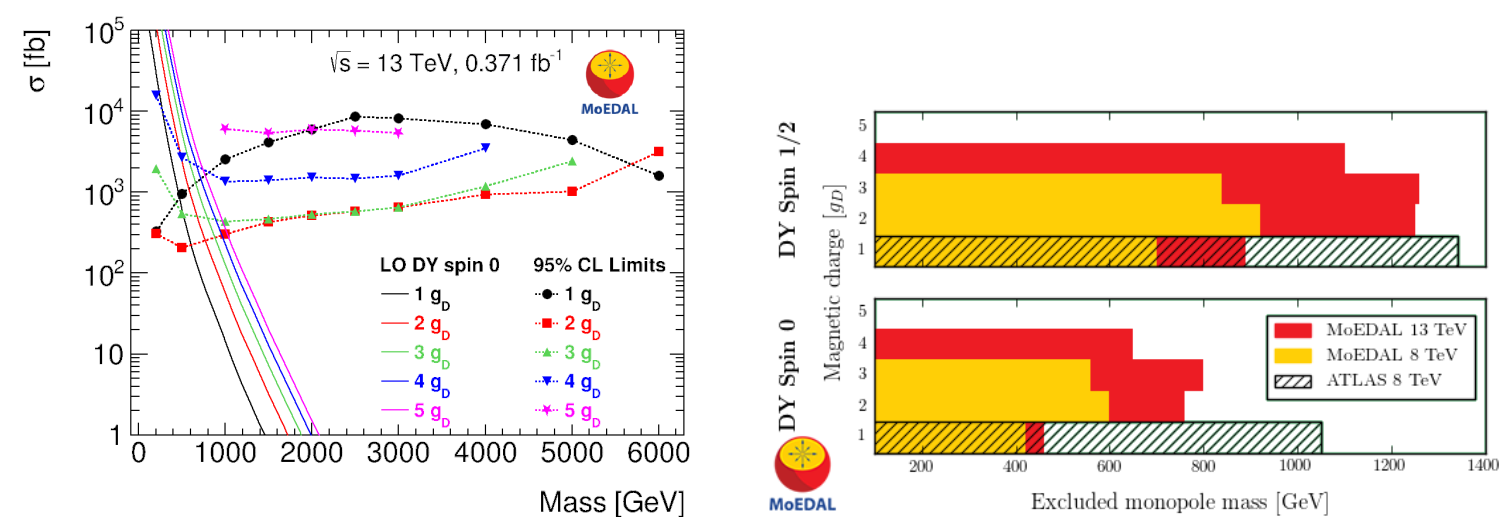

Figure 4: Left: Cross-section upper limits at 95\% confidence level for DY monopole production as a function of mass for spin- 0 monopoles. The various line styles correspond to different monopole charges. The solid lines represent DY cross-section calculations at leading order [85]. Right: Excluded monopole masses for DY production for spin-1/2 (top) and spin-0 (bottom) monopoles. The MoEDAL results obtained at $8 \mathrm{TeV}$ (yellow, light grey) [84] and $13 \mathrm{TeV}$ (red, dark grey) [85] are superimposed on the ATLAS 8-TeV limits (hatched area) [78].

\section{Outlook}

The existence of magnetic monopoles, if confirmed experimentally, would modify our understanding of electrodynamics, rendering the Maxwell equation fully symmetric. The Dirac quantisation condition is a beautiful consequence of the existence of monopoles and therefore they represent an extremely appealing physical scenario. Various searches have beed carried out utilising diverse detection techniques in both observational facilities and experiments in colliders.

Neutrino telescopes, such as ANTARES and IceCube currently, and KM3NeT and PINGU in the future, pursue the search for GUT monopoles. In addition, an astroparticle extension to MoEDAL is proposed keeping the same detector technology [87]. Cosmic-MoEDAL proposal contemplates a $50000-100000 \mathrm{~m}^{2}$ of plastic NTDs deployed at high altitude. Such an array would be able to take the search for cosmic monopoles with velocities $\beta \gtrsim 0.1$ from the TeV scale to the GUT scale for monopole fluxes well below the Parker bound.

NOvA is a long-baseline neutrino experiment designed to study neutrino oscillations in the Fermilab NuMI beam. The far detector, located at the surface in Minnesota, near the U.S.-Canada border, comprises liquid scintillator cells read out at both ends by avalanche photodiodes. This detector has the potential to cover a still unexplored phase-space region of intermediate-mass slow monopoles of $10^{-4} \lesssim \beta \lesssim 0.2$ due to its location on the surface and large surface area. To this effect, a dedicated data-driven trigger for slow monopoles has been designed [88].

The Iron CALorimeter (ICAL) at the India-based Neutrino Observatory (INO), aiming to measure neutrino oscillation parameters, is also sensitive to sub-relativistic magnetic monopoles [89]. Using ICAL, a possible monopole event will be characterised by the large time intervals of up to $0.3 \mu \mathrm{s}$ between the signals in successive layers of the active tracking detectors. In the mass range $10^{7}-10^{17} \mathrm{GeV}$ and for velocities $\beta \sim 0.001-0.7$, the detector sensitivity is $\sim 1.6 \times$ $10^{-16} \mathrm{~cm}^{-2} \mathrm{~s}^{-1} \mathrm{sr}^{-1}$ for a live-time of 10 years. The sensitivity can be increased by a factor of two with some additional detectors on the walls of the cavern. 
The CERN LHC, being the most powerful collider to-date, is the ideal machine to produce magnetic monopoles, if they exist. In particular, the MoEDAL experiment is specialising in the detection of magnetic monopoles and other highly ionising particles. MoEDAL has set the stringent bounds on high magnetic charges and together with other LHC experiments, such as ATLAS, are going to continue probing the existence of light monopoles. Moreover, the possibility of analysing decommissioned parts of the LHC beam-pipe system at the ATLAS, CMS and LHCb/MoEDAL sites with a SQUID to search for trapped magnetic monopoles has been proposed [90]. Another possibility to probe magnetic monopoles, is given by their bound state, the monopolium, through their multiphoton final states $[18,19]$ that can be tested in ATLAS and CMS.

\section{Acknowledgments}

The author is grateful to the Corfu2017 organisers for the kind invitation to present this talk. She acknowledges support by the Generalitat Valenciana through the MoEDAL-supporting agreement CON.21.2017-09.02.03 and the Excellence Project PROMETEO-II/2017/033, by the Spanish MINEICO under the project FPA2015-65652-C4-1-R, by the Severo Ochoa Excellence Centre Project SEV-2014-0398, and by a 2017 Leonardo Grant for Researchers and Cultural Creators, BBVA Foundation.

\section{References}

[1] P. A. M. Dirac, Proc. Roy. Soc. Lond. A 133, 60 (1931).

[2] G. 't Hooft, Nucl. Phys. B 79, 276 (1974); A. M. Polyakov, JETP Lett. 20, 194 (1974) [Pisma Zh. Eksp. Teor. Fiz. 20, 430 (1974)].

[3] B. Julia and A. Zee, Phys. Rev. D 11, 2227 (1975); Y. Nambu, Nucl. Phys. B 130, 505 (1977); E. Witten, Phys. Lett. 86B, 283 (1979); G. Lazarides, M. Magg and Q. Shafi, Phys. Lett. 97B, 87 (1980); R. d. Sorkin, Phys. Rev. Lett. 51, 87 (1983); D. J. Gross and M. J. Perry, Nucl. Phys. B 226, 29 (1983); J. S. Schwinger, Science 165, 757 (1969); J. Preskill, Ann. Rev. Nucl. Part. Sci. 34, 461 (1984); A. Achucarro and T. Vachaspati, Phys. Rept. 327, 347 (2000) [Phys. Rept. 327, 427 (2000)]; T. W. Kephart, C. A. Lee and Q. Shafi, JHEP 0701, 088 (2007); D. G. Pak, P. M. Zhang and L. P. Zou, Int. J. Mod. Phys. A 30, no. 27, 1550164 (2015); A. Rajantie, JHEP 0601, 088 (2006); T. W. Kephart, G. K. Leontaris and Q. Shafi, JHEP 1710, 176 (2017).

[4] Y. M. Cho and D. Maison, Phys. Lett. B 391, 360 (1997); W. S. Bae and Y. M. Cho, J. Korean Phys. Soc. 46, 791 (2005).

[5] P. A. M. Dirac, Phys. Rev. 74, 817 (1948).

[6] A. Rajantie, Contemp. Phys. 53, 195 (2012).

[7] A. Rajantie, Phys. Today 69, no. 10, 40 (2016).

[8] V. Vento and V. S. Mantovani, On the magnetic monopole mass, arXiv:1306.4213 [hep-ph] (2013).

[9] M. Barriola and A. Vilenkin, Phys. Rev. Lett. 63, 341 (1989).

[10] P. O. Mazur and J. Papavassiliou, Phys. Rev. D 44, 1317 (1991); N. E. Mavromatos and J. Papavassiliou, Eur. Phys. J. C 78, no. 1, 68 (2018).

[11] A. K. Drukier and S. Nussinov, Phys. Rev. Lett. 49, 102 (1982). 
[12] N. E. Mavromatos and S. Sarkar, Phys. Rev. D 95, no. 10, 104025 (2017); "Regularised Kalb-Ramond Magnetic Monopole with Finite Energy,” Phys. Rev. D, to appear [arXiv:1804.01702 [hep-th]] (2018).

[13] Y. M. Cho, K. Kimm and J. H. Yoon, Mod. Phys. Lett. A 31, no. 09, 1650053 (2016); Phys. Lett. B 761, 203 (2016).

[14] J. Ellis, N. E. Mavromatos and T. You, Phys. Lett. B 756, 29 (2016).

[15] Y. B. Zeldovich and M. Y. Khlopov, Phys. Lett. 79B, 239 (1978).

[16] C. T. Hill, Nucl. Phys. B 224, 469 (1983); V. K. Dubrovich, Grav. Cosmol. Suppl. 8N1, 122 (2002).

[17] L. N. Epele, H. Fanchiotti, C. A. Garcia Canal and V. Vento, Eur. Phys. J. C 56, 87 (2008); Eur. Phys. J. C 62, 587 (2009).

[18] L. N. Epele, H. Fanchiotti, C. A. G. Canal, V. A. Mitsou and V. Vento, Eur. Phys. J. Plus 127, 60 (2012); L. N. Epele, H. Fanchiotti, C. A. G. Canal, V. A. Mitsou and V. Vento, Can the $750 \mathrm{GeV}$ enhancement be a signal of light magnetic monopoles?, arXiv:1607.05592 [hep-ph] (2016).

[19] H. Fanchiotti, C. A. García Canal and V. Vento, Int. J. Mod. Phys. A 32, no. 35, 1750202 (2017).

[20] C. Patrignani et al. [Particle Data Group], Chin. Phys. C 40, no. 10, 100001 (2016).

[21] S. Cecchini, L. Patrizii, Z. Sahnoun, G. Sirri and V. Togo, Energy Losses of Magnetic Monopoles in Aluminum, Iron and Copper, arXiv:1606.01220 [physics.ins-det] (2016).

[22] V. A. Rubakov, JETP Lett. 33, 644 (1981) [Pisma Zh. Eksp. Teor. Fiz. 33, 658 (1981)]; V. A. Rubakov and M. S. Serebryakov, Nucl. Phys. B 218, 240 (1983); C. G. Callan, Jr., Nucl. Phys. B 212, 391 (1983).

[23] M. S. Turner, E. N. Parker and T. J. Bogdan, Phys. Rev. D 26, 1296 (1982).

[24] F. C. Adams, M. Fatuzzo, K. Freese, G. Tarle, R. Watkins and M. S. Turner, Phys. Rev. Lett. 70, 2511 (1993).

[25] B. Cabrera, Phys. Rev. Lett. 48, 1378 (1982).

[26] M. E. Huber, B. Cabrera, M. A. Taber and R. D. Gardner, Phys. Rev. Lett. 64, 835 (1990).

[27] P. B. Price, E. K. Shirk, W. Z. Osborne and L. S. Pinsky, Phys. Rev. Lett. 35, 487 (1975).

[28] J. M. Kovalik and J. L. Kirschvink, Phys. Rev. A 33, 1183 (1986); H. Jeon and M. J. Longo, Phys. Rev. Lett. 75, 1443 (1995) Erratum: [Phys. Rev. Lett. 76, 159 (1996)].

[29] P. B. Price, S. 1. Guo, S. P. Ahlen and R. L. Fleischer, Phys. Rev. Lett. 52, 1265 (1984).

[30] L. Patrizii and M. Spurio, Ann. Rev. Nucl. Part. Sci. 65, 279 (2015).

[31] M. Ambrosio et al. [MACRO Collaboration], Nucl. Instrum. Meth. A 486, 663 (2002).

[32] M. Ambrosio et al. [MACRO Collaboration], Eur. Phys. J. C 25, 511 (2002).

[33] S. Orito et al., Phys. Rev. Lett. 66, 1951 (1991).

[34] Y. F. Novoseltsev, M. M. Boliev, A. V. Butkevich, S. P. Mikheev and V. B. Petkov, Nucl. Phys. Proc. Suppl. 151, 337 (2006).

[35] J. L. Thron et al. [Soudan-2 Collaboration], Phys. Rev. D 46, 4846 (1992).

[36] M. R. Krishnaswamy et al., Phys. Lett. 142B, 99 (1984). 
[37] S. Balestra et al., Eur. Phys. J. C 55, 57 (2008).

[38] K. Antipin et al. [BAIKAL Collaboration], Astropart. Phys. 29, 366 (2008).

[39] R. Abbasi et al., Eur. Phys. J. C 69, 361 (2010).

[40] S. Adrian-Martinez et al. [ANTARES Collaboration], Astropart. Phys. 35, 634 (2012).

[41] A. Albert et al. [ANTARES Collaboration], JHEP 1707, 054 (2017).

[42] R. Abbasi et al. [IceCube Collaboration], Phys. Rev. D 87, no. 2, 022001 (2013).

[43] M. G. Aartsen et al. [IceCube Collaboration], Eur. Phys. J. C 76, no. 3, 133 (2016).

[44] P. Sapienza [KM3NeT Collaboration], Nucl. Part. Phys. Proc. 291-293, 183 (2017).

[45] M. G. Aartsen et al. [IceCube Collaboration], J. Phys. G 44, no. 5, 054006 (2017).

[46] M. G. Aartsen et al. [IceCube Collaboration], Eur. Phys. J. C 74, no. 7, 2938 (2014).

[47] F. Lauber [IceCube Collaboration], Magnetic monopole searches with IceCube, $6^{\text {th }}$ International Conference on New Frontiers in Physics (ICNFP 2017), 17-26 August 2017, Kolymbari, Crete, Greece.

[48] A. Aab et al. [Pierre Auger Collaboration], Phys. Rev. D 94, no. 8, 082002 (2016).

[49] D. P. Hogan, D. Z. Besson, J. P. Ralston, I. Kravchenko and D. Seckel, Phys. Rev. D 78, 075031 (2008).

[50] M. Detrixhe et al. [ANITA-II Collaboration], Phys. Rev. D 83, 023513 (2011).

[51] J. E. Bartelt et al., Phys. Rev. D 36, 1990 (1987) Erratum: [Phys. Rev. D 40, 1701 (1989)].

[52] M. Ambrosio et al. [MACRO Collaboration], Eur. Phys. J. C 26, 163 (2002).

[53] R. Becker-Szendy et al., Phys. Rev. D 49, 2169 (1994).

[54] V. A. Balkanov et al. [Baikal Collaboration], Prog. Part. Nucl. Phys. 40, 391 (1998).

[55] K. Ueno et al. [Super-Kamiokande Collaboration], Astropart. Phys. 36, 131 (2012).

[56] A. De Rujula, Nucl. Phys. B 435, 257 (1995); I. F. Ginzburg and A. Schiller, Phys. Rev. D 60, 075016 (1999).

[57] B. Abbott et al. [D0 Collaboration], Phys. Rev. Lett. 81, 524 (1998).

[58] M. Acciarri et al. [L3 Collaboration], Phys. Lett. B 345, 609 (1995).

[59] L. P. Gamberg, G. R. Kalbfleisch and K. A. Milton, Difficulties with photonic searches for magnetic monopoles, hep-ph/9805365 (1998).

[60] O. Gould and A. Rajantie, Phys. Rev. Lett. 119, no. 24, 241601 (2017)

[61] A. Abulencia et al. [CDF Collaboration], Phys. Rev. Lett. 96, 201801 (2006).

[62] G. R. Kalbfleisch, W. Luo, K. A. Milton, E. H. Smith and M. G. Strauss, Phys. Rev. D 69, 052002 (2004).

[63] P. B. Price, G. X. Ren and K. Kinoshita, Phys. Rev. Lett. 59, 2523 (1987).

[64] B. Aubert, P. Musset, M. Price and J. P. Vialle, Phys. Lett. 120B, 465 (1983).

[65] R. A. Carrigan, F. A. Nezrick and B. P. Strauss, Phys. Rev. D 8, 3717 (1973).

[66] G. Abbiendi et al. [OPAL Collaboration], Phys. Lett. B 663, 37 (2008). 
[67] J. L. Pinfold, R. Du, K. Kinoshita, B. Lorazo, M. Regimbald and B. Price, Phys. Lett. B 316, 407 (1993).

[68] K. Kinoshita, R. Du, G. Giacomelli, L. Patrizii, F. Predieri, P. Serra, M. Spurio and J. L. Pinfold, Phys. Rev. D 46, R881 (1992).

[69] K. Kinoshita, M. Fujii, K. Nakajima, P. B. Price and S. Tasaka, Phys. Lett. B 228, 543 (1989).

[70] P. Musset, M. Price and E. Lohrmann, Phys. Lett. 128B, 333 (1983).

[71] T. Gentile et al. [CLEO Collaboration], Phys. Rev. D 35, 1081 (1987).

[72] W. Braunschweig et al. [TASSO Collaboration], Z. Phys. C 38, 543 (1988).

[73] A. Aktas et al. [H1 Collaboration], Eur. Phys. J. C 41, 133 (2005).

[74] L. Evans and P. Bryant, JINST 3, S08001 (2008).

[75] G. Aad et al. [ATLAS Collaboration], JINST 3, S08003 (2008).

[76] J. Pinfold et al. [MoEDAL Collaboration], Technical Design Report of the MoEDAL Experiment, CERN-LHCC-2009-006, MoEDAL-TDR-001; MoEDAL web page: http://moedal.web.cern.ch/

[77] G. Aad et al. [ATLAS Collaboration], Phys. Rev. Lett. 109, 261803 (2012).

[78] G. Aad et al. [ATLAS Collaboration], Phys. Rev. D 93, 052009 (2016).

[79] T. Akesson et al., Nucl. Instrum. Meth. A 474, 172 (2001); M. Aaboud et al. [ATLAS Collaboration], JINST 12, no. 05, P05002 (2017).

[80] A. De Roeck, A. Katre, P. Mermod, D. Milstead and T. Sloan, Eur. Phys. J. C 72, 1985 (2012).

[81] M. Fairbairn, A. C. Kraan, D. A. Milstead, T. Sjostrand, P. Z. Skands and T. Sloan, Phys. Rept. 438, 1 (2007); S. Burdin, M. Fairbairn, P. Mermod, D. Milstead, J. Pinfold, T. Sloan and W. Taylor, Phys. Rept. 582, 1 (2015).

[82] B. Acharya et al. [MoEDAL Collaboration], Int. J. Mod. Phys. A 29, 1430050 (2014).

[83] A. De Roeck, H. P. Hächler, A. M. Hirt, M.-D. Joergensen, A. Katre, P. Mermod, D. Milstead and T. Sloan, Eur. Phys. J. C 72, 2212 (2012).

[84] B. Acharya et al. [MoEDAL Collaboration], JHEP 1608, 067 (2016).

[85] B. Acharya et al. [MoEDAL Collaboration], Phys. Rev. Lett. 118, 061801 (2017).

[86] B. Acharya et al. [MoEDAL Collaboration], Phys. Lett. B, to appear, doi:10.1016/j.physletb.2018.05.069 [arXiv:1712.09849 [hep-ex]] (2018).

[87] J. L. Pinfold [MoEDAL Collaboration], EPJ Web Conf. 126, 02024 (2016).

[88] Z. Wang, Search for Magnetic Monopoles with the NOvA Far Detector, PhD thesis, University of Virginia, FERMILAB-THESIS-2015-28, doi:10.2172/1230047 (2015).

[89] N. Dash, V. M. Datar and G. Majumder, Astropart. Phys. 70, 33 (2015).

[90] A. De Roeck, P. Mermod, J. Pinfold et al., Searching for trapped magnetic monopoles in LHC accelerator material, https://indico.cern.ch/event/623746/attachments/1427507/2190853/ expression_of_interest_beam_pipe.pdf 